\title{
Grating influence study of GaAs solar cell structures
}

\author{
S. Saravanan, R. S. Dubey \\ Department of Nanotechnology, Swarnandhra College of Engineering and Technology, Seetharampuram, \\ Narsapur-534280, (A.P.), India \\ rag_pcw@yahoo.co.in
}

DOI 10.17586/2220-8054-2017-8-4-503-506

\begin{abstract}
Thin film solar cells are having the problem of low absorption of light, particularly at longer wavelengths and hence, efficient light trapping engineering is demanded. Here, we propose a design of ultra thin GaAs solar cell with enhanced light absorption with the use of dual (dielectric and metal) gratings. In this way, light trapping can be enhanced at longer wavelengths for both TE and TM polarization modes.
\end{abstract}

Keywords: solar cells, ultra thin, GaAs, dielectric, metal, polarization mode.

Received: 28 September 2016

Revised: 20 May 2017

\section{Introduction}

Presently, GaAs solar cells have shown high power conversion efficiency relative to other solar cell technologies. Among semiconductor materials, GaAs has the better optical performance, e.g. high energy photons can be easily absorbed in short region, less power consumption, high crystal quality, and operating frequency. Additionally, various theoretical and experimental light trapping mechanisms have been reported for the better harvesting of light with the use of nanograting, nanoparticles and back reflectors etc. [1-3]. Hong et al. have reported designing GaAs thin film solar cells by incorporating a silver nanoparticles in a periodical manner. They have observed enhanced absorption due to the surface plasmon induced by metal nanoparticles. GaAs based solar cells could result in $31 \%$ improvement in short-circuit current as compared to a planar solar cell [4]. Nakayama et al. have experimentally demonstrated an improvement in GaAs solar cells by using silver nanoparticles fabricated by masked deposition. They have observed strong scattering by the interacting surface plasmons and increased optical path for the incident photons which could give an $8 \%$ increase in the short-circuit current density [5]. Chang et al. have presented a modeling of GaAs solar cells using the finite-difference time domain (FDTD) method. The fabricated device consisted of self-assembled two-dimensional microspheres and was found to be efficient for light harvesting. Comparison of the designed solar cell with the fabricated one could show a $25 \%$ enhanced conversion efficiency [6]. Grandidier et al. have demonstrated the design of perfectly flat GaAs solar cell structures of varying thicknesses that were comprised of a double layer antireflection coating, silica nanosphere array on top and a back reflector. The solar cell with $100 \mathrm{~nm}$ thickness showed better improvement however, in the case of the cell having $1000 \mathrm{~nm}$ thickness, a $2.5 \%$ improvement was observed with the optimization of the sphere size and the spacing between them [7]. Zhang et al. have presented a study of light trapping properties of GaAs nanoneedle arrays based solar cells by employing rigorous coupled wave analysis and finite element method. They compared nanowire array-based solar cells with thin film layer based and observed enhanced light absorption. This enhancement has been attributed to the graded refractive index of nanoneedle arrays that could couple the incident light in an efficient manner and also was observed to be less dependent on the incident angle. With an optimized solar cell structure, they observed enhanced absorption, as much as more than $90 \%$ compared to the band gap [8].

In this paper, we present a modeling and simulation of GaAs solar cells based on the dual gratings. In section 2, the design approach with simulation details is presented and results are discussed in section 3. Finally, section 4 concludes the paper.

\section{Design approach}

The schematic diagram of the solar cell structure with proposed light trapping mechanism is depicted in Fig. 1. This design architecture consists of $70 \mathrm{~nm}$ anti-reflection coating (ARC) of silicon nitride $\left(\mathrm{Si}_{3} \mathrm{~N}_{4}\right)$, top dielectric grating (height $30 \mathrm{~nm}$ ) of indium-tin-oxide, bottom metal grating (height $30 \mathrm{~nm}$ ) of aluminum, $40 \mathrm{~nm}$ GaAs active layer and $150 \mathrm{~nm}$ aluminum (Al) substrate. Here, the use of dielectric and metal gratings produce multiple bouncings of the incident photons in the active region which prolongs optical path length or lifetime of the photons.

Modeling of the proposed structure was investigated by using finite-difference-time-domain method, which deals with Maxwell's equations of electromagnetic waves. For the simulation, the periodic boundary conditions 


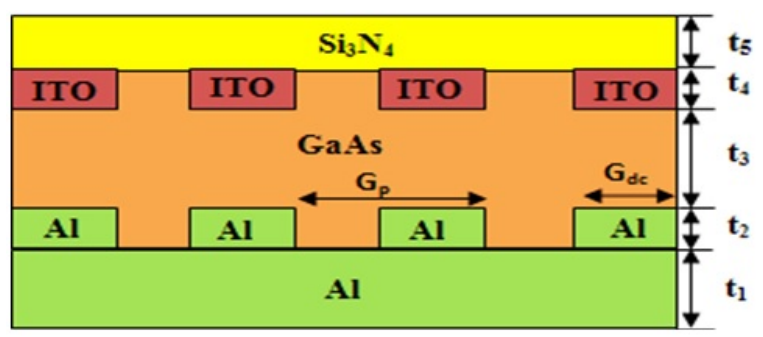

FIG. 1. Schematic diagram of proposed GaAs solar cell

were applied in $\mathrm{x}$ - and $\mathrm{y}$-directions while a perfectly matched layer condition was applied in the $\mathrm{z}$-direction to prevent the reflections. In this work, the FDTD method was employed to analyze the electric and magnetic field distributions in the proposed solar cell and results will be explored in the next section.

However, the performance of solar cells was analytically evaluated by employing the basic characteristics equations. The expressions of short-circuit current $\left(J_{s c}\right)$ and open-circuit voltage $V_{o c}$ can be expressed as:

$$
\begin{gathered}
J_{s c}=e N_{e-h}\left[\frac{A}{m^{2}}\right], \\
V_{o c}=\frac{k T}{e} \ln \left(\frac{J_{s c}}{J_{s o}}+1\right)[V],
\end{gathered}
$$

where $k$ is the Boltzmann's constant, $T$ is the absolute temperature and $J_{s o}$ is the reverse-bias saturation current.

Using the short-circuit current and open-circuit voltage equations (1) and (2), one can write an expression of fill factor $(F F)$ as:

$$
F F=\frac{J_{m p} \times V_{m p}}{J_{s c} \times V_{o c}}
$$

Therefore, fill factor is defined as the ratio of maximum power and product of short-circuit current and open-circuit voltage.

By using equations (1), (2) and (3), one can evaluate the solar cell efficiency $(\eta)$ with the following expression:

$$
\eta=\frac{J_{s c} \times V_{o c} \times F F}{P_{i n}}
$$

where $P_{i n}$ is the total incident power.

The above resultant equation is used to evaluate the performance of the solar cell as a function of short-circuit current, open-circuit voltage, fill factor and the incident power.

\section{Results and Discussion}

For a comparative study of the light absorption in the proposed dual grating based solar cell (named as D), we have designed three solar cells; A: reference solar cell without grating, B: with only bottom grating and C: with only top grating. Fig. 2(a,b) shows absorption curves of various solar cells for both the transverse electric (TE) and magnetic (TM) polarizations respectively. Refer to Fig. 2(a) for the TE case, optimal enhancement in light absorption can be observed for the dual grating based solar cell however, reference solar cell shows poor absorption from the wavelength $600 \mathrm{~nm}$. The enhanced absorption from the solar cell $\mathrm{D}$ has been attributed to the scattering effect of metal and guiding effect of dielectric gratings respectively. Fig. 2(b) shows absorption of various designed solar cells for the TM case. In the case of reference solar cell, low absorption can be observed while dual grating based solar cell shows optimal absorption over a broad range of the wavelength region due to the induced plasmonic effect.

For the case of bottom grating based design, localized surface plasmon and guided modes are observed. Generally, the metallic grating structure excites localized surface plasmon resonance which prolongs the optical path length of photons in the absorbing layer [1,9]. In the case of solar cell $\mathrm{D}$, the combined effect of dual grating supported by plasmonic and photonic modes is observed. We have calculated the relative enhancement of short-circuit current density of the solar cell D with reference to the solar cell A by using an expression (Cell $D_{J s c}-$ Cell $A_{J s c} /$ Cell $\left.A_{J s c}\right)$. A relative enhancement in short-circuit current density about 101.6 and $216.7 \%$ are obtained for TE and TM respectively. The use of either top or bottom grating shows enhanced absorption as compared to the reference solar cell A. However, the performance of solar cell D is dominant, which is due to the light trapping phenomena. 

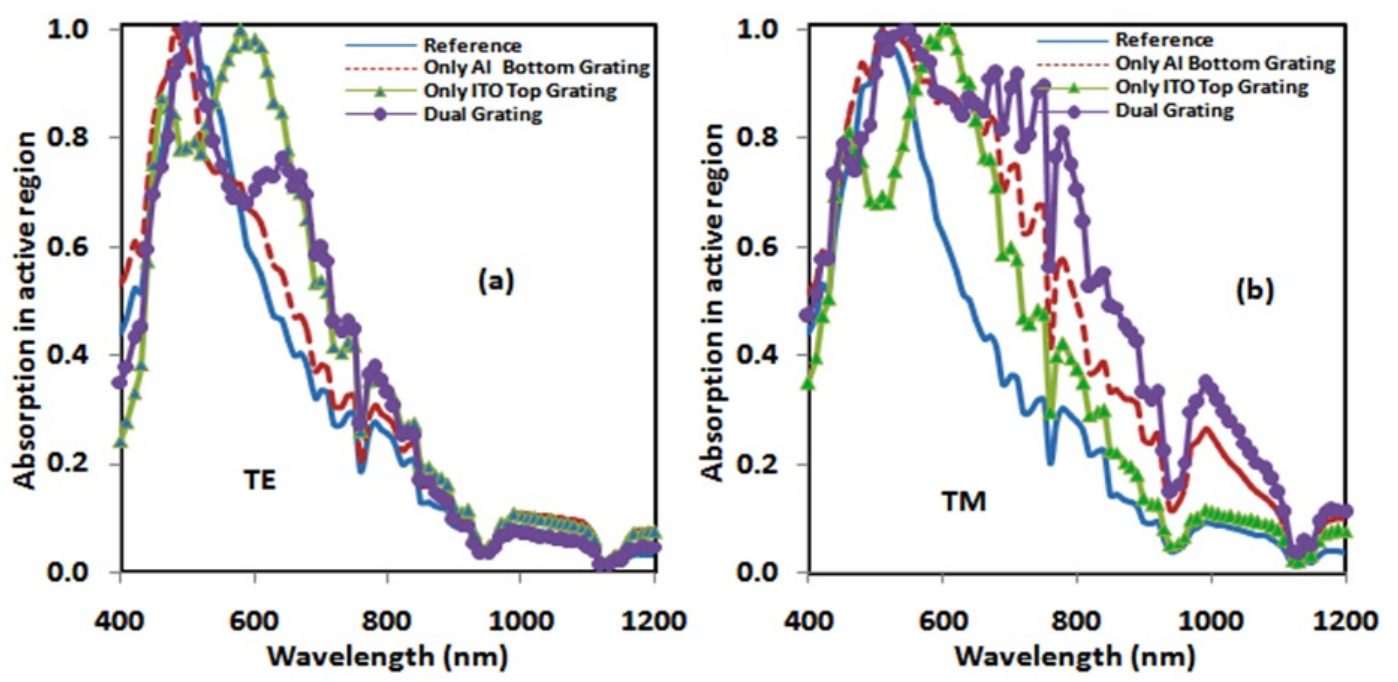

FIG. 2. Light absorption for TE Fig.(a) and TM Fig.(b) cases

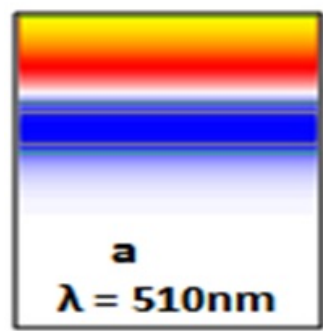

TE polarization case
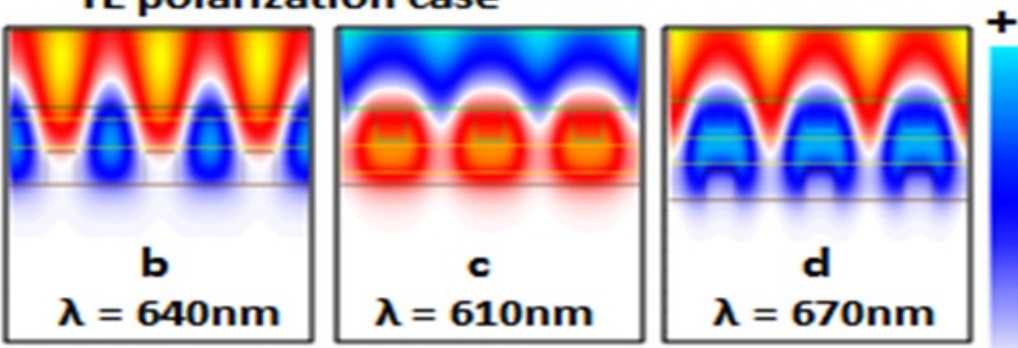

TM polarization case
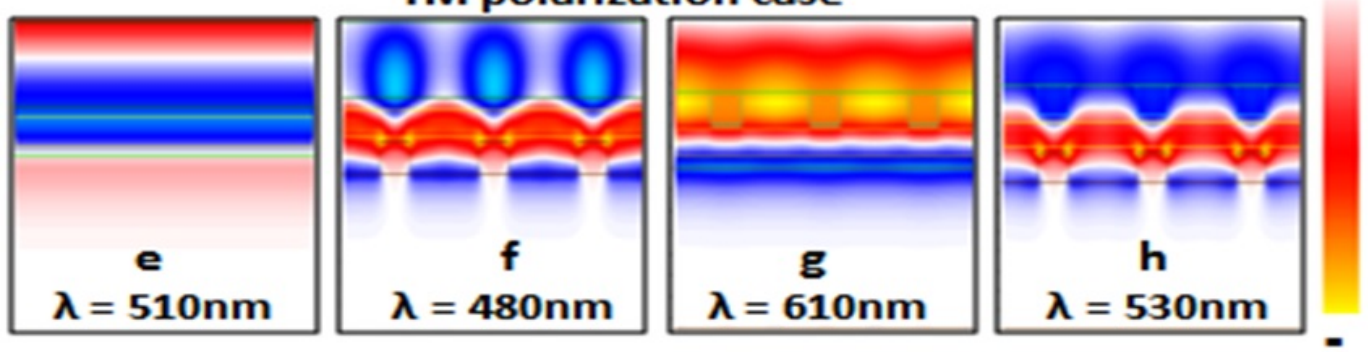

FIG. 3. Transverse electric and magnetic field distributions in different solar cell structures

After collecting the peak values of different solar cell designs from absorption curves plotted in Fig. 2, we have studied the field profile for both the cases of TE and TM polarizations which is shown in Fig. 3. The usual behavior of field distribution can be seen in Fig. 3(a,e) for the case of reference solar cell A. Also, in Fig. 3(b-d) and Fig. 3(f-h) an improvement in field profile is also observed from different solar cells based on bottom, top and dual gratings in reference to solar cells B, C and D respectively. However, a distinct field profile is observed for the transverse magnetic field mode. With the solar cell D, as shown in Fig. 3(h) the localized surface plasmon (LSP) supported by the guided modes can be observed. This magnetic field distribution behavior is responsible for the enhanced cell efficiency.

\section{Conclusion}

We have investigated the optical performance of ultrathin dual grating GaAs solar cell and compared that with a reference solar cell. Among other designed solar cells, dual grating based solar cell could yield maximum shortcircuit current density 17.41 for TE and $29.66 \mathrm{~mA} / \mathrm{cm}^{2}$ for the TM case. This enhancement has been attributed to the combined effect of photonic and plasmonic modes. Finally, a relative enhancement in short-circuit current density about 101.6 and $216.7 \%$ has been obtained for TE and TM respectively. 


\section{Acknowledgments}

The financial support from DRDO is acknowledged.

\section{References}

[1] Ronen Chriki, Avner Yanai, Joseph Shappir and Uriel Levy. Enhanced efficiency of thin film solar cells using a shifted dual grating plasmonic structure. Opt. Express, 2013, 21(S3), P. A381-A391.

[2] Yanpeng Shi, Xiaodong Wang, Wen Liu, Tianshu Yang, Rui Xu, and Fuhua Yang. Multilayer silver nanoparticles for light trapping in thin film solar cells. J. Appl. Phys., 2013, 113, P. 176101-1-3.

[3] Sangjun Lee and Sangin Kim. Optical absorption characteristic in thin a-Si film embedded between an ultrathin metal grating and a metal reflector. IEEE Photon. J., 2013, 5(5), P. 4800610-1-9.

[4] Lei Hong, Rusli, Xincai Wang, Hongyu Zheng, Lining He, Xiaoyan Xu et. al. Design principles for plasmonic thin film GaAs solar cells with high absorption enhancement. J.Appl. Phys., 2012, 112, P. 054326-1-5.

[5] Keisuke Nakayama, Katsuaki Tanabe and Harry A. Atwater. Plasmonic nanoparticle enhanced light absorption in GaAs solar cells. Appl.Phys.Lett., 2008, 93, P. 121904-1-3.

[6] Te-Hung Chang, Pei-Hsuan Wu, Sheng-Hui Chen, Chia Hua Chan, Cheng-Chung Lee et. al. Efficiency enhancement in GaAs solar cells using self-assembled microspheres. Opt. Express, 2009, 7(9), P. 6519-6524.

[7] Jonathan Grandier, Callahan Dennis M., Munday Jeremy N. and Atwater Harry A. Gallium arsenide solar Cell absorption enhancement using whispering gallery modes of dielectric nanospheres. IEEE J. Photovolt., 2012, 2(2), P. 123-128.

[8] Xu Zhang, Xiao-Hong Sun and Liu-Di Jiang. Absorption Enhancement Using Nanoneedle Array for Solar Cell. Appl. Phys. Lett., 2013, 103, P. 211110-1-5.

[9] Saravanan S., Dubey R.S., Kalainathan S., More M.A. and Gautam D.K. Design and optimization of ultrathin crystalline silicon solar cells using an efficient back reflector. $A I P A d v$., 2015, 5, P. 057160-1-9. 